\title{
Quantitative evaluation of the impact of heterogeneity correction on left breast cancer radiotherapy performed with respiratory gating
}

\author{
Mohamed Taher Fdhila1,2, Isabelle Gabelle-Flandin'1, Jacques Balosso ${ }^{1,2}$, Abdulhamid Chaikh ${ }^{1,2}$ \\ ${ }^{1}$ Department of Radiation Oncology and Medical Physics, University Hospital of Grenoble, France \\ ${ }^{2}$ University of Grenoble-Alpes, Grenoble, France
}

Received July 07, 2015; Revised December 29, 2015; Accepted December 31, 2015; Published Online January 03, 2016

Original Article

\begin{abstract}
Purpose: Our objective was to assess the impact of a heterogeneity correction to the calculated dose for left breast cancer gated radiotherapy. Methods: Ten patients with left breast cancer were studied. For each patient 2 treatment plans were generated. In plan 1 the dose was calculated using a Pencil Beam Convolution (PBC) algorithm. In plan 2 the dose was calculated using the Modified Batho's (MB) density correction method. To compare the two plans a dosimetric analysis was carried out including monitor units (MU), isodose curves, cumulative and differential dose volume histograms (cDVH, dDVH), coverage index, conformity index for target volume and the two dimensional (2D) gamma index $(\gamma)$. Wilcoxon signed rank and Spearmen's tests were used to calculate $p$-values and correlation coefficients (r), respectively. Results: MB method reduced the MU by on average $1.12 \pm 5.33 \%$. The analysis of $\mathrm{cDVH}$ showed that the MB method calculated significantly higher doses for target volumes, lung and heart, $p<0.05$. The data demonstrated a strong correlation between the dosimetric parameters derived from plan 1 and plan 2 with $r>0.9$. The 2D $\gamma$ analysis showed that the difference between plan 1 and plan 2 could reach $\pm 10 \%$. The $\gamma$ evaluation showed a high impact of density correction for left breast cancer with gating technique. Conclusion: This study confirms that using the MB method integrated with a PBC algorithm, the calculated dose will be increased to target volumes, lung and heart. Even more so since gating usually tends to decrease average lung density by about $39 \%$ by treating during an arrested inspiration phase. Thus, attention should be paid when changing from PBC to newer algorithms with gating techniques, since the probability of cardiac mortality and lung toxicity are correlated to absorbed dose.
\end{abstract}

Keywords: Heterogeneity Correction for Breast; Gating Method

\section{Introduction}

Recent advances in radiotherapy have introduced several irradiation techniques for breast cancer. Several studies have demonstrated that respiratory gating improves the accuracy of dose delivery to the target volume in breast cancer radiotherapy by reducing doses to organs at risk (OAR), i.e the heart and lungs. ${ }^{1,2}$ During the treatment, the dose received by the target volume (TV) should be close to the prescribed dose. The former is currently predicted by the treatment planning

software based on dose calculation models. Certain organs i.e. lung and heart have tolerance dose limits that should be respected and an accurate dose calculation is necessary for this. Presently, there are various algorithms and methods to take account of the heterogeneity correction of tissues. Numerous reviews in the literature have categorized the heterogeneity correction methods according to various criteria. Recently, Chaikh et al. classified the algorithms into

\footnotetext{
Corresponding author: Abdulhamid Chaikh; Department of Radiation Oncology and Medical Physics, University Hospital of Grenoble,

France.
}

Cite this article as: Fdhila MT, Gabelle-Flandin I, Balosso J, Chaikh A. Quantitative evaluation of the impact of heterogeneity correction on left breast cancer radiotherapy performed with respiratory gating. Int J Cancer Ther Oncol 2016; 4(1):417.

DOI: 10.14319/ijcto.41.7 
three types according to whether they take into account, or not, the density correction and inclusion or exclusion of electron transport. ${ }^{3}$

- Type (a): change in lateral transport of electrons is not modelled, e.g. density correction methods integrated into the pencil beam convolution algorithm (PBC) in the Eclipse ${ }^{\circledR}$ treatment planning system (TPS) (Varian Medical Systems, Palo Alto, CA), such as Modified Batho's (MB) density correction method.

- Type (b): approximate the transport of electrons such as the anisotropic analytical algorithm (AAA) and collapsed cone convolution (CCC) implemented respectively in the Eclipse and Pinnacle $^{\circledR}$ (Philips Radiation Oncology Systems, Fitchburg, WI) TPS.

- Type (c): which solves the linear Boltzmann transport equation: Acuros XB (Varian Medical Systems, Inc., Palo Alto, CA).

Type (a) and type (b) algorithms calculate absorbed dose to water. In contrast, Acuros XB a type (c), calculates dose to a medium, which can be converted into the dose to water for treatment plan evaluation. When the beam goes through relatively low-density lung tissue, the heterogeneity correction should be done with an accurate algorithm. Recent studies have discussed the impact of low-density organs on the coverage of adjacent target volumes, as in breast cancer treatment. They showed that the dose calculation algorithm has a significant impact on dosimetry and radiobiological model parameters. ${ }^{4-8}$ However, we have little knowledge about the impact of heterogeneity corrections on target volume and organs at risk using the gating technique for breast cancer. In the present study, we will assess what the impact would be of switching from $\mathrm{PBC}$ to $\mathrm{MB}$ on doses when using gating technique radiotherapy.

\section{Methods and Materials}

\subsection{Clinical cases and treatment planning}

This study is based on ten patients with left breast cancer. These cases were chosen to cover the full range of the different types of radiotherapy, namely: breast alone, breast with boost, breast with internal mammary chain (IMC) or with IMC and the supra-clavicular area. A computed tomography (CT-scan) was done using the respiratory gating technique. Then the images were loaded into the TPS.

The virtual simulation for each patient was generated by a digitally reconstructed radiograph (DRR) and beam's eye view (BEV) information. In this way a treatment field was superimposed on the DRR to assess target localization. The target volumes including the security margins and the organs at risk were delineated by the radiation oncologist. The treatment was performed with four tangential beams according to the technique currently used in our department: 2 tangential beams of 6 Megavoltage (MV) and 2 tangential beams of $18 \mathrm{MV}$. The combination of four beams of two energies with different beam ponderation and field size, with or without a wedge, has been introduced to optimize the dose homogeneities more accurately than with just a pair of tangential beams with wedge filters. The conventional radiation course varied from 46.91 to 50.6 Gy in 23 - 25 daily fractions of 1.87 - 2.2 Gy on the whole breast followed by the addition of a boost to the tumor bed. The boost consisted of doses varying from 11 to 15 Gy in 5 - 23 daily fractions of 0.5 to 2.5 Gy.

For each patient, two treatment plans were generated: in plan 1 the dose was calculated using the PBC which was taken as the reference dose, in plan 2 the dose was calculated using the MB method in combination with the PBC algorithm. The PBC algorithm is a convolution algorithm based on pencil beam kernels. First, the dose distribution in a homogeneous water equivalent medium is calculated. Next, the tissue densities are taken into account by multiplying the dose distribution by a correction factor. In the MB method, the inhomogeneity correction factor is calculated along the central axis.

\subsection{Treatment plans evaluations}

\subsubsection{Monitor units (MU)}

A calculation of the average and standard deviation was performed for each field.

\subsubsection{Isodose curves}

The $95 \%$ and $100 \%$ isodose curves encompassing the TV were compared.

\subsubsection{Dose volume histogram (DVH)}

For each TV the minimum dose (Dmin), mean dose (Dmean), maximum dose (Dmax) and the calculated dose to $95 \%$ of the target volume (D95\%) were compared using cumulative cDVH. For lung and heart the Dmin, Dmean and Dmax were compared. The dose homogeneity inside the TV, lung and heart were compared using a S-index associated with the differential dDVH ${ }^{9}$ :

$$
S-\text { index }=\sqrt{\frac{\sum_{\mathrm{j}=1}^{\mathrm{TV}}(\mathrm{D}(\mathrm{j})-\text { Dmean })^{2}}{\mathrm{TV}}}
$$

where, $D(j)$ is the relative dose in the lesion voxel $j$, Dmean is the average relative dose in the lesion and TV is the target volume in elementary voxels.

For each patient, to compare plan 2 with plan 1, the calculated doses in plan 1 were taken as the reference values. The difference in percentage was calculated as:

$$
\Delta \text { Dose }(\%)=\left(D_{M B}-D_{P B C}\right) \times 100 / D_{M B}
$$




\subsubsection{Quality indices}

The coverage index (CI) and conformity index for the target volume (CITV) proposed by the Radiation Therapy Oncology Group (RTOG) were compared using the following equations 10,11 :

$$
\mathrm{CI}=\frac{\mathrm{Imin}}{\mathrm{RI}}
$$

where, Imin is the minimal isodose surrounding the target and RI is the reference isodose.

$$
\text { CITV }=\frac{\text { Volume receiving 95\% of PD }}{\mathrm{TV}}
$$

where, PD is the prescribed dose and TV is the target volume.

\subsubsection{Variation of lung density using a gating technique}

The CT images using either free breathing or the gating technique were exported to TPS, then the average hounsfield unit (HU) value was calculated for lung. Next, The HU was converted to relative electronic density $\left(\rho_{\mathrm{w}}\right)$ using the following equation ${ }^{12}$ :

$$
\mathrm{HU}=1000 \cdot\left[\left(\rho / \rho_{\mathrm{w}}\right)-1.0\right]
$$

where, $\rho_{w}$ is the density relative to water measured using CT images.

Figure 1 shows an example of a HU calculation using the TPS for a volume of interest located in the lung.

\subsubsection{Gamma index}

The impact of heterogeneity correction was analyzed using the two dimensional (2D) gamma index $(\gamma)$ by comparing dose distributions from plan 1 and plan 2.13, $\mathbf{1 4 , 1 5}$ Our goal was to determine the volume ratio receiving the same irradiation, in terms of $\gamma$ which combines two criteria including the dose difference $(\triangle D o s e)$ in percentage and the distance-to -agreement (DTA) in millimeters. An ellipse is used to determine the acceptable region, and $\gamma \leq 1$ represents fulfillment of the criteria. The DICOM images for each patient were exported from TPS to RIT-113 ${ }^{\circledR}$ (Dosimetry System Version 5.2, Radiological Imaging Technology, Inc., CO) including the dose distributions. The dimensions were $20 \times 20 \mathrm{~cm}^{2}$ with a resolution of $0.39 \mathrm{~mm}$. The results were displayed using $\gamma$ maps and dose pixel histograms (DPH). The $\gamma$ maps show the pixels with $\gamma$ values greater than unity that were outside of tolerance range. The pixels with $\gamma>1$ show either an under- or overestimated dosage associated with plan 2 compared to plan 1 . In order to discriminate between an over- and an under-estimated dose, a color-code was attributed to the $\triangle$ Dose. The DPH indicates the fraction of pixels with a specific value of $\Delta$ Dose. Using the $\gamma$ criteria $(3 \%, 3 \mathrm{~mm})$ we considered that the plan 1 and plan 2 were similar if $95 \%$ of pixels had $\gamma \leq 1$.

\subsubsection{Statistical analysis}

A Wilcoxon signed rank test was used to calculate the $p$-value. A bilateral statistical test was realized with an error $\alpha=5 \%$, corresponding to a $95 \%$ confidence interval. The $\Delta$ dose is considered significant if $p<0.05$. Data are presented as average \pm standard deviation (SD). The statistical correlation between calculated doses from plan 1 and plan 2 was evaluated using Spearmen's correlation coefficient. ${ }^{16}$ The correlation between density difference ( $\Delta$ density) and $\Delta$ dose was also evaluated.

\section{Results}

\subsection{Monitor units}

The MB reduced the MU by on average $1.12 \pm 5.33 \%$. The Wilcoxon test showed that there was no significant difference between plan 1 and plan 2, $p=0.27$ and the data showed a strong correlation with $r=0.99$.

\subsection{Isodose curves}

In the transversal plans, we observed that both the $95 \%$ and $100 \%$ isodose curves encompassed the same fraction of the TV with the two techniques. The $40 \%$ isodose curve is closer to the heart and lung with $\mathrm{MB}$ than with PBC. Figure 2 shows isodose curves in color shading showing the dose distribution from plan 1 with PBC and plan 2 with MB.

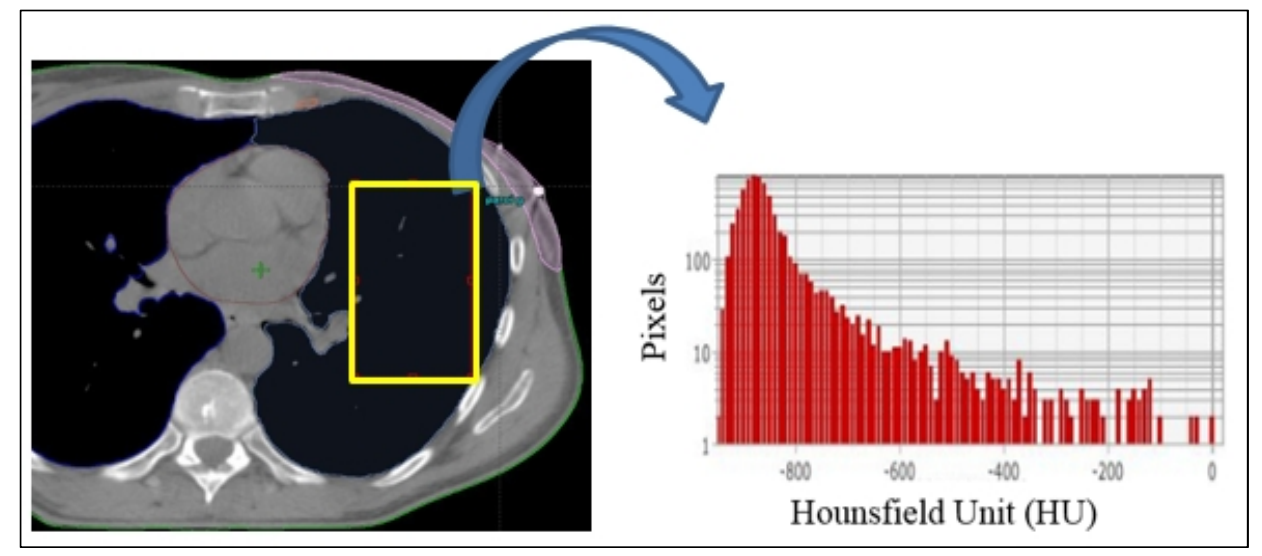

Figure 1: Example of a HU calculation using the TPS for a volume of interest located in the lung. 


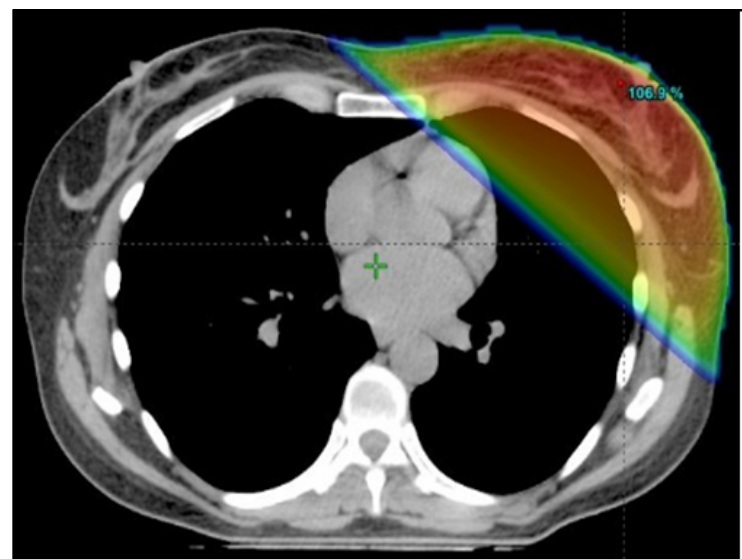

(A)

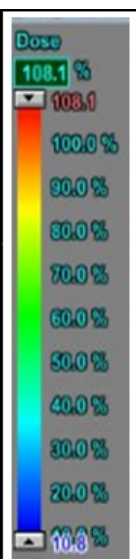

Figure 2: Dose distribution according to plan (A) with PBC and (B) with MB.

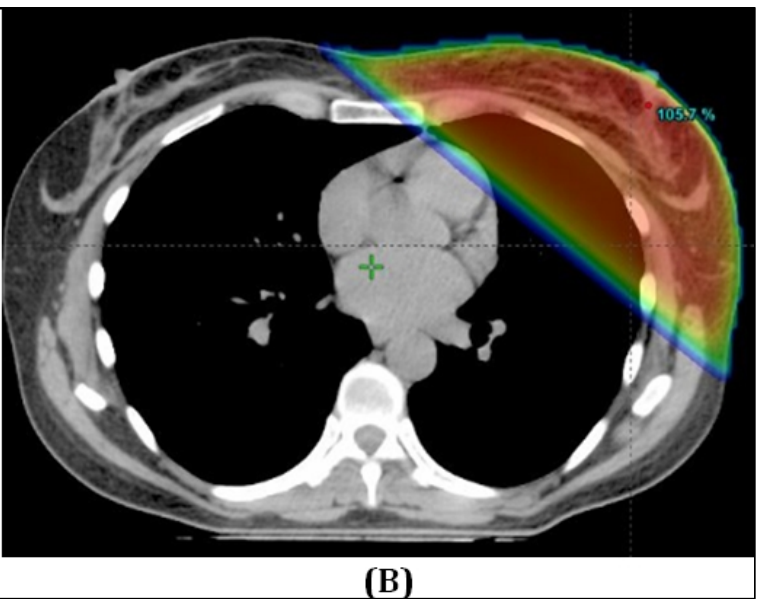

(B)

\subsection{Dose volume histograms}

\subsection{1 cDVH}

Figure 3 shows the cDVH from plan 1 and plan 2. The Tables 1 and 2 show dosimetric and statistical results obtained for TV, lung and heart. In Table 1, it can be seen that the Wilcoxon test indicated a very significant difference between calculated doses with PBC and MB.
Spearman's test indicated a strong correlation for Dmin, Dmean and D95\%. In Table 2, it can be seen that MB calculated higher doses for lung and heart with very significant differences, except for the lung's Dmin. The Spearman's test indicated a strong correlation except for the lung's Dmax.

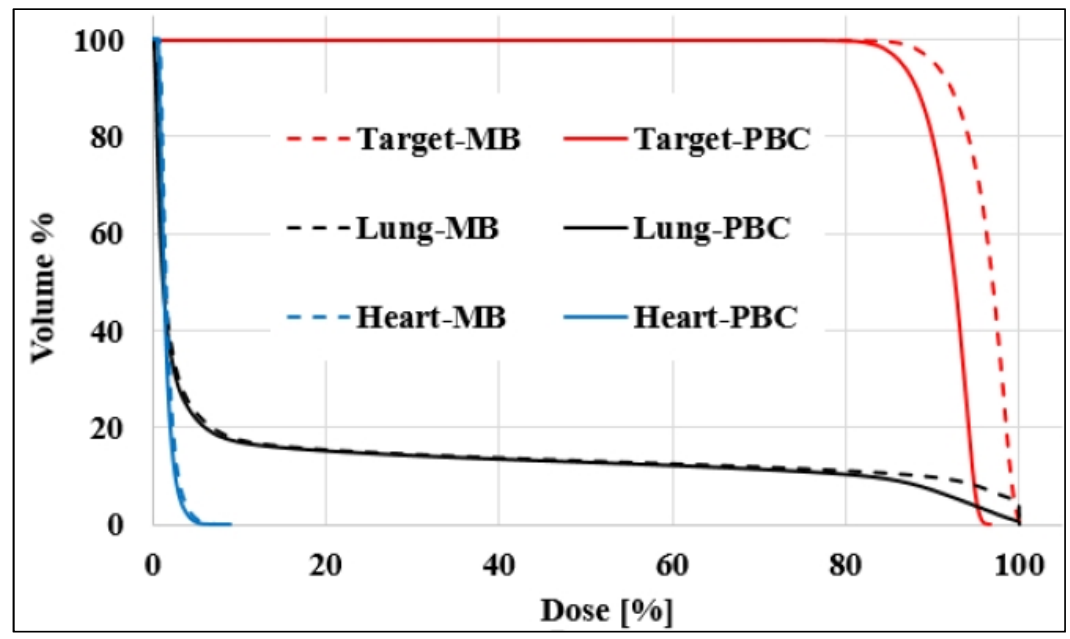

Figure 3: Cumulative dose volume histograms calculated using the PBC algorithm without heterogeneity correction for plan 1 , and the MB method with 1D heterogeneity correction for plan 2.

Table 1: Dose differences for dose volume histograms for target volumes.

\begin{tabular}{ccccc}
\hline \hline$\Delta$ Dose $\%$ & Dmin & Dmean & Dmax & D95\% \\
\hline Average \pm SD & $3.88 \pm 6.3$ & $2.55 \pm 3.11$ & $2.71 \pm 3.45$ & $2.27 \pm 6.48$ \\
r-value & 0.99 & 0.97 & 0.77 & 0.9 \\
$p$-value & $<0.01$ & $<0.001$ & $<0.001$ & $<0.001$ \\
\hline \hline
\end{tabular}

Table 2: Dose differences for dose volume histograms for organs at risk.

\begin{tabular}{ccccc}
\hline \hline & $\Delta$ Dose $\%$ & Dmin & Dmean & Dmax \\
\hline Lung & Average \pm SD & $5.8 \pm 12.4$ & $7.6 \pm 1.3$ & $3.5 \pm 1.7$ \\
& r-value & 0.93 & 0.99 & 0.52 \\
& $p$-value & 0.37 & $<0.01$ & $<0.01$ \\
\hline Heart & Average \pm SD & $23 \pm 8.5$ & $11.41 \pm 4.48$ & $8.35 \pm 4.29$ \\
& r-value & 0.94 & 0.98 & 0.99 \\
& $p$-value & $<0.01$ & $<0.01$ & $<0.01$ \\
\hline \hline
\end{tabular}




\subsection{2 dDVH}

The dose distribution inside the TV, lung and heart using MB was slightly more heterogeneous than with PBC. The average S-indexes for the target were $10.52 \pm 10.11$ and $10.37 \pm 10.04$ for $\mathrm{MB}$ and $\mathrm{PBC}$, respectively. Wilcoxon and Spearman's tests showed no-significant differences but strong correlations with $p=0.46$ and $r=0.99$. For lung, the average S-indexes were $31.3 \pm 4.19$ and $29.28 \pm$ 4.04 for MB and PBC, respectively. For heart, the average S-indexes were $1.89 \pm 1.99$ and $1.81 \pm 2.01$ respectively. Wilcoxon and Spearmen's tests showed a significant difference and strong correlation with $p<0.005$ and $\mathrm{r}=$ 0.99 .

\subsection{Quality indices}

The average CIs were $1.05 \pm 0.58$ and $0.98 \pm 0.56$ for $\mathrm{MB}$ and $\mathrm{PBC}$, respectively, with $p=0.03$ and $r=0.98$. The average CITVs were $0.67 \pm 0.27$ and $0.63 \pm 0.29$ for $\mathrm{MB}$ and $\mathrm{PBC}$, respectively, with $p=0.20$ and $\mathrm{r}=0.81$. Figure 4 shows the indices values using a boxplot representation. It can be seen that both plan 1 and 2 have the same median values for $\mathrm{CI}$ and close values for CITV.

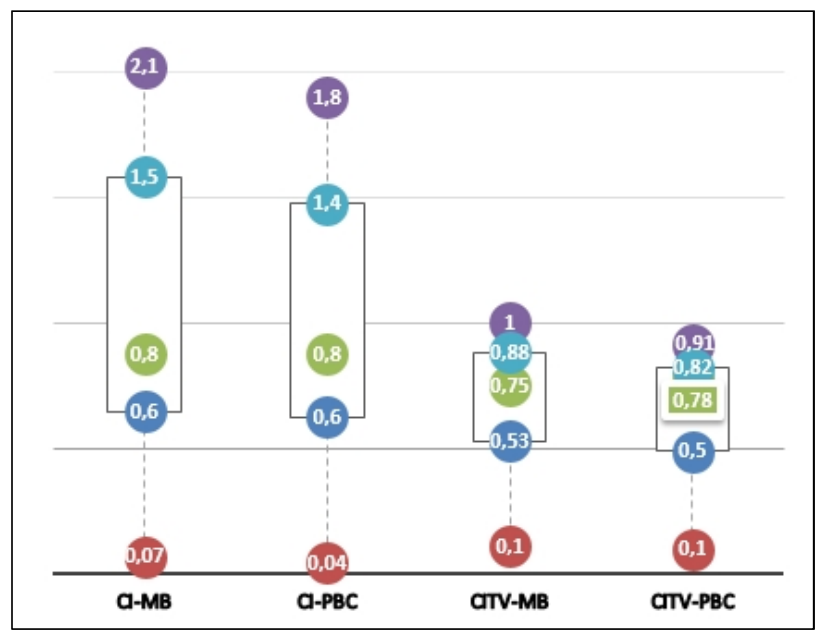

Figure 4: Boxplot for quality indices indicating the minimum, median and maximum values, as well as the $25^{\text {th }}$ and $75^{\text {th }}$ percentiles.

\subsection{Variation of lung density using a gating technique}

The HU calculated for lung was $-727.25 \pm 42$ and -835.25 \pm 23.68 using free breathing and gating techniques, respectively. This leads to a decrease in lung density with the gating technique from $0.27 \pm 0.04$ to $0.16 \pm$ 0.02 . The Wilcoxon test showed a very significant decrease of density with $p<0.001$. However, the data for density are highly variable from one patient to another, which explains why no correlation appears between the density and the associated breathing modality, free breathing or gating, $r=0.36$. This is due to basal tissue density and a morphologic effect resulting in the gating technique being very different for each patient.

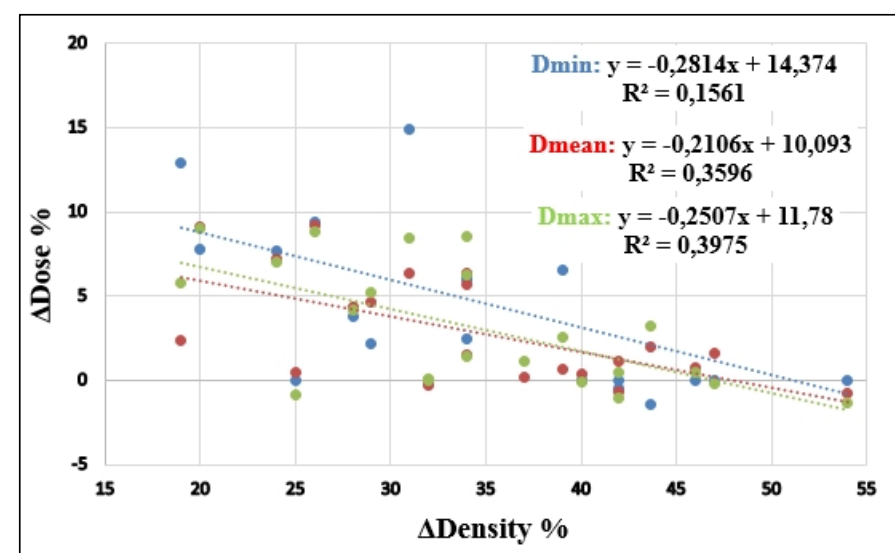

Figure 5: Correlation between $\Delta$ density and $\Delta$ dose for target volume in all patients.

Lung inflation during an inspiration, with gating increases the lung volume, which will consequently increase the distance between the TV and the heart. However, there was a poor correlation between density differences ( $\Delta$ density) and $\Delta$ dose, with $\mathrm{r}$ values ranging from $r=0.52$ to 0.62 . Figure 5 shows the correlation between density and dose for Dmin, Dmean and Dmax for the TV for all patients.

\subsection{D gamma}

The average maximum and mean $\gamma$ values were $2.82 \pm$ 0.65 and $0.23 \pm 0.11$, respectively. The tolerance limit of $95 \%$ of pixels having $\gamma \leq 1$ is not respected at all. The average $\gamma$ passing rate was $92.5 \% \pm 4.98 \%$. Figure 6 shows an example of a 2D $\gamma$ map in the axial plane. The red and blue coloring indicate when the $\gamma$ value $>1$ and identify overestimated dose $\left(\mathrm{D}_{\mathrm{MB}}>\mathrm{D}_{\mathrm{PBC}}\right)$ or underestimated dose $\left(\mathrm{D}_{\mathrm{MB}}<\mathrm{D}_{\mathrm{PBC}}\right)$, respectively. Figure 7 shows the DPH obtained from plan 1 and plan 2. It can be seen that the tolerance limit of $95 \%$ of pixels having $\gamma$ $\leq 1$ can be satisfied with a $\Delta$ Dose $=7 \%$ and $3 \mathrm{~mm}$. The correlation test showed that there was a weak correlation between $\gamma$ passing rates and dose difference.
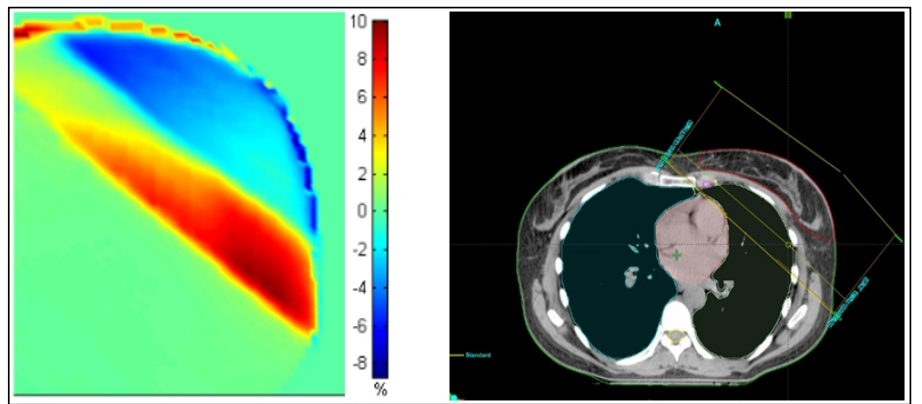

Figure 6: An example of a 2D $\gamma$-map plotted in the axial plane (left panel) and CT-scan (right panel) corresponding to treatment for one patient with four tangential fields. 


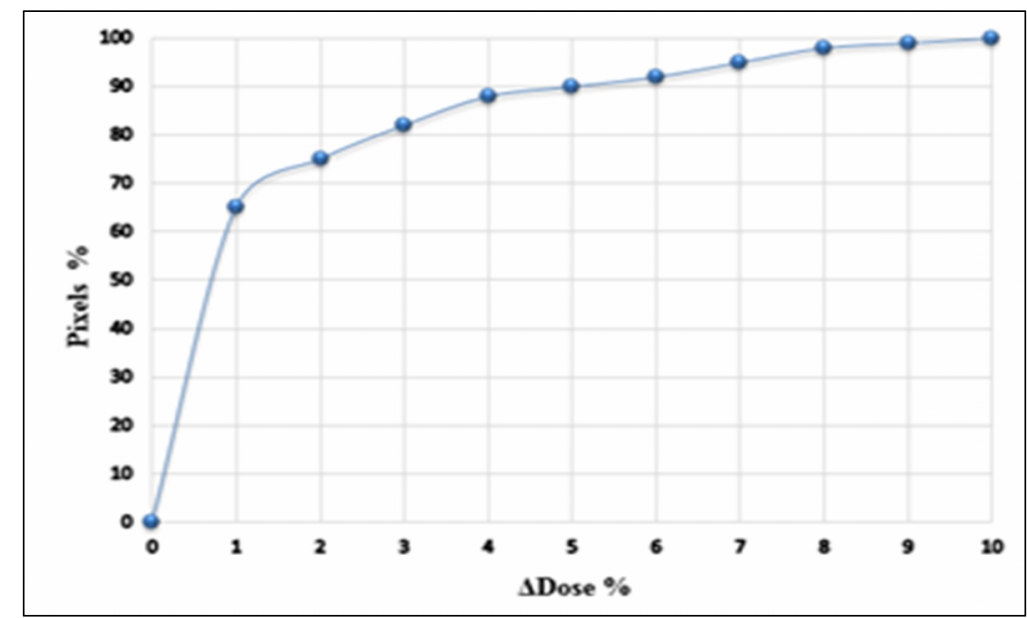

Figure 7: DPH obtained with MB and PBC from plan 1 and plan 2 respectively.

\section{Discussion}

Using the respiratory gating technique, we observed that the lung density was decreased by $38.7 \pm 9.82 \%$. The range effect of lateral electrons is expected to be larger if the lung density is lower. The MB method integrated with PBC does not model the lateral electron transport. This algorithm will overestimate the coverage of the TV and underestimate the low dose volumes in or near to OAR. In this study, the MU for the set of treatments was substantially shorter with MB than with PBC, $p>0.05$. The cDVH and dDVH analysis for the TV showed that MB overestimated the dose for the target volume with a significant difference, $p<0.05$ (as shown in Table 1). Identifying the TV locations, we observed that the significant differences, up to $12 \%$, were located in the internal mammary chain areas.

This study confirms, using the MB algorithm, that dosimetric parameters, derived from cDVH to lung and heart, will be increased (as shown in Table 2). The 2D $\gamma$ analysis showed that the difference between plan 1 and plan 2 could reach up to $\pm 10 \%$. Therefore, the tolerance level of $95 \%$ pixels with $\gamma \leq 1$ was not respected, showing a higher impact when using the density correction. Since respiratory gating is intended to reduce the dose to the heart and lungs, with the aim of reducing the risk of cardiac mortality and lung toxicity, one should take account of the alterations in dosimetry introduced by this technique, to retain its advantages. Thus, any change from PBC to newer algorithms, i.e. CCC, AAA or Acuros $\mathrm{XB}$, and even more so in the context of respiratory gating, should be very carefully undertaken. ${ }^{17-24}$

\section{Conclusion}

The most recent algorithms approximate lateral electron transport accurately, either in breast tissue with a density close to water, or lung with lower density. When using the gating technique for breast cancer treatment the lung density will be significantly decreased from 0.27 to $0.16, p<0.001$. The MB method for $1 \mathrm{D}$ heterogeneity correction overestimates the dose to the heart and lung. The physicist should be aware of the differences between the different dose calculation algorithms and should be careful when changing from older algorithms such as PBC to newer algorithms, in order to optimally protect the heart and lung.

\section{Conflict of interest}

The authors declare that they have no conflicts of interest. The authors alone are responsible for the content and writing of the paper.

\section{Acknowledgement}

We thank Dr. Alison Foote (Grenoble University Hospital) for language editing.

\section{References}

1. Korreman SS, Pedersen AN, Nottrup TJ, et al. Breathing adapted radiotherapy for breast cancer: comparison of free breathing gating with the breath-hold technique. Radiother Oncol. 2005;76:311-8.

2. Korreman SS, Pedersen AN, Josipovic M, et al. Cardiac and pulmonary complication probabilities for breast cancer patients after routine end-inspiration gated radiotherapy. Radiother Oncol. 2006; 80: 257-62.

3. Chaikh A, Balosso J. Should the dose prescription be readjusted when using tissues density corrections algorithms for radiation oncology? J Case Rep Onc Ther. 2014; 1:01018.

4. Petillion S, Swinnen A, Defraene G, et al. The photon dose calculation algorithm used in breast radiotherapy has significant impact on the parameters of radiobiological models. J Appl Clin Med Phys. 2014;15:4853. 
5. Basran PS, Zavgorodni S, Berrang T, et al. The impact of dose calculation algorithms on partial and whole breast radiation treatment plans. Radiat Oncol. 2010;5:120.

6. Fogliata A, Nicolini G, Clivio A, Vanetti E, Cozzi L. On the dosimetric impact of inhomogeneity management in the Acuros XB algorithm for breast treatment. Radiat Oncol. 2011;6:103.

7. Yoo S, Wu Q, O'Daniel J, et al. Comparison of 3D conformal breast radiation treatment plans using the anisotropic analytical algorithm and pencil beam convolution algorithm. Radiother Oncol. 2012;103:172-7.

8. Edvardsson A, Nilsson MP, Amptoulach S, Ceberg S. Comparison of doses and NTCP to risk organs with enhanced inspiration gating and free breathing for left-sided breast cancer radiotherapy using the AAA algorithm. Radiat Oncol. 2015;10:84.

9. Yoon M, Park SY, Shin D, et al. A new homogeneity index based on statistical analysis of the dose-volume histogram. J Appl Clin Med Phys. 2007;8:9-17.

10. Feuvret L, Noël G, Mazeron JJ, Bey P. Conformity index: a review. Int J Radiat Oncol Biol Phys. 2006;64:333-42.

11. Chaikh A, Giraud J, Balosso J. A method to quantify and assess the dosimetric and clinical impact resulting from the heterogeneity correction in radiotherapy for lung cancer. Int J Cancer Ther Oncol. 2014; 2:020110.

12. Gurjar OP, Mishra SP, Bhandari V, et al. Radiation dose verification using real tissue phantom in modern radiotherapy techniques. J Med Phys. 2014;39:44-9.

13. Low DA, Harms WB, Mutic S, Purdy JA. A technique for the quantitative evaluation of dose distributions. Med Phys. 1998; 25: 656-61.
14. Spezi E, Lewis DG. Gamma histograms for radiotherapy plan evaluation. Radiother Oncol. 2006;79:224-30.

15. Chaikh A, Giraud J, Balosso J. A 3D quantitative evaluation for assessing the changes of treatment planning system and irradiation techniques in radiotherapy. Int J Cancer Ther Oncol. 2014; 2:02033.

16. Chaikh A, Giraud JY, Perrin E, et al. The choice of statistical methods for comparisons of dosimetric data in radiotherapy. Radiat Oncol. 2014;9:205.

17. Papanikolaou N, Battista J, Mackie T, Kappas C, Boyer A. Tissue inhomogeneity corrections for megavoltage photon beams. AAPM Report No 85; Task Group No. 65, 2004.

18. Ahnesjö A, Aspradakis MM. Dose calculations for external photon beams in radiotherapy. Phys Med Biol. 1999; 44:99-155.

19. Batho HF. Lung corrections in cobalt 60 beam therapy. J Can Assoc Radiol. 1964;15:79-83.

20. El-Khatib E, Battista JJ. Improved lung dose calculation using tissue-maximum ratios in the Batho correction. Med Phys.1984;11:279-86.

21. Thomas SJ. A modified power low formula for inhomogeneity corrections in beams of high energy x-rays. Med Phys. 1991;18:719-23.

22. Rana S. Clinical dosimetric impact of Acuros XB and analytical anisotropic algorithm (AAA) on real lung cancer treatment plans: review. Int J Cancer Ther Oncol. 2014;2:02019.

23. Ojala J. The accuracy of the Acuros XB algorithm in external beam radiotherapy - a comprehensive review. Int J Cancer Ther Oncol. 2014;2:020417.

24. Rana S, Pokharel S. Dose-to-medium vs. dose-to-water: Dosimetric evaluation of dose reporting modes in Acuros XB for prostate, lung and breast cancer. Int J Cancer Ther Oncol. 2014;2:020421. 